\title{
Storage Efficiency Optimization of a Supercapacitor Charged by a Photovoltaic Cell using Genetic Algorithm
}

\author{
Sachin Seth ${ }^{1}$, Sudipto Mukherjee ${ }^{2}$, Tanusree Dutta ${ }^{3}$, Rabindranath Ghosh ${ }^{4}$ \\ Dept. of ECE, St. Thomas' College of Engineering \& Tech., Kolkata, India ${ }^{1}$ \\ Dept. of ECE, St. Thomas' College of Engineering \& Tech, Kolkata, India ${ }^{2}$ \\ Assistant Professor, ECE, St. Thomas' College of Engineering \& Tech, Kolkata, India ${ }^{3}$ \\ Professor, ECE, St. Thomas' College of Engineering \& Tech, Kolkata, India ${ }^{4}$
}

\begin{abstract}
Super capacitors are very power dense devices in which charge density depends on the value of the capacitance and voltage across it. Generally, high capacitance of the Supercapacitor would support higher energy storage density, voltage remaining fixed. The load driven also does not utilize properly the entire stored charge from the Super capacitor due to inconsistency in electrical characteristics between the load and source. Storage efficiency of Super capacitor has state of charge dependencies as it is variable over the duration of charge/discharge cycles. It depends directly on the capacitance and indirectly on the ESR value. The proposed optimization technique can significantly find the maximum value of storage efficiency at a near about minimum value of ESR and maximum value of capacitance. The simulation model and results show the advantage of the said technique.
\end{abstract}

Keywords: Supercapacitor, Photovoltaic panel, RC branch model, Energy storage system, Solar photovoltaic equation, Genetic algorithm, Storage efficiency, Perturb \& Observe algorithm, Buck Converter.

\section{INTRODUCTION}

Solar energy is one of the most widely available sources of renewable energy. It's abundance in availability and consistent supply from our sun makes it one of the most promising source of energy to drive our future energy needs. The most common devices used to convert this energy are photovoltaic (PV) cells [1]. Alternatives exist but photovoltaic being produced in mass scale reduces the cost of manufacturing and also has very low maintenance costs. They generate no pollution and there is almost zero

Greenhouse gas emissions. The efficiency of photovoltaic cells is low (10-25\%) which occasionally can reach $35 \%$ and for this reason tracking of the incident sunlight is necessary in order to extract maximum power from the PV panels [2]. Many methods are present and one such efficient method is the Maximum Point tracking Algorithm (MPPT). As the intensity of solar radiation varies, the load characteristics that provide the best power transfer efficiency change and as a result maximum power extraction is affected. The MPPT keeps track on the changes in load characteristics to obtain the maximum power point. Maximum power point trackers may employ various algorithms such as Incremental conductance, Perturb \& Observe ( $\mathrm{P} \& \mathrm{O})$, Constant Voltage, Current sweep etc. Among the mentioned algorithms the $\mathrm{P} \& \mathrm{O}$ algorithm shows the best performance [3] [6]. MPPT algorithms change the impedance of the circuit by employing DC-DC converters controlled by the MPPT controller so as to maximize the power extraction from the

PV panels to the load [4]. Load in most of the cases is usually a battery as they are the most widely used storage devices and are great in storing electrical energy. However they take large time to store electrical energy in the form of chemical energy and also they lack the ability to transfer power quickly to the load. They cannot handle bursts of power in applications such as torque generation or regenerative braking in motors of electric vehicles. To overcome such problems Supercapacitors are used which are basically hybrid of capacitors and batteries. Unlike batteries they can charge or discharge energy at a very fast rate similar to a normal capacitor but their energy storage capacity is much larger compared to a normal capacitor similar to a battery.

They have large charging and discharging cycles compared to rechargeable batteries. They generally use electrostatic double layer capacitance and electrochemical pseudo capacitance with variable contribution of each [5]. Supercapacitor have large storage efficiencies. These are one of the most efficient storage devices. Storage efficiency of Supercapacitors is generally a function of the internal resistance also called as equivalent series resistance (ESR) and the capacitance. The storage efficiency is directly proportional to the capacitance value and inversely proportional to the internal resistance ESR. 
Our main area of interest in this paper is to find optimum values of ESR and Capacitance which would maximize the storage efficiency of the Supercapacitor. In this work we have used Genetic algorithm which is an AI based searching technique to optimize the values of ESR and Capacitance so as to get the maximum possible efficiency.

The organization of this paper is as follows. In the next part (part II) a detailed description of the mathematical and electrical models used in the simulation is discussed and also the various algorithms used in these work. Part III verifies the facts and part IV provides the results obtained from our simulation and observation. Conclusion is given in Part V.

\section{MATHEMATICAL MODELS AND ALGORITHMS}

\section{A. Model of the Photo-voltaic panel}

Photovoltaic or solar cells are devices which are usually in the form of thin films or wafers. They are semiconductor devices with large p-n junctions usually made of $\mathrm{Si}$, that convert from 3-30\% of incident solar energy to DC electricity, with efficiencies depending on illumination-spectrum intensity, solar-cell design and materials and temperature. It generally behaves like a low-voltage battery whose charge is continuously replenished at a rate proportional to incident illumination. Connection of such cells into series-parallel configurations allows the design of solar panels with high current and voltages.

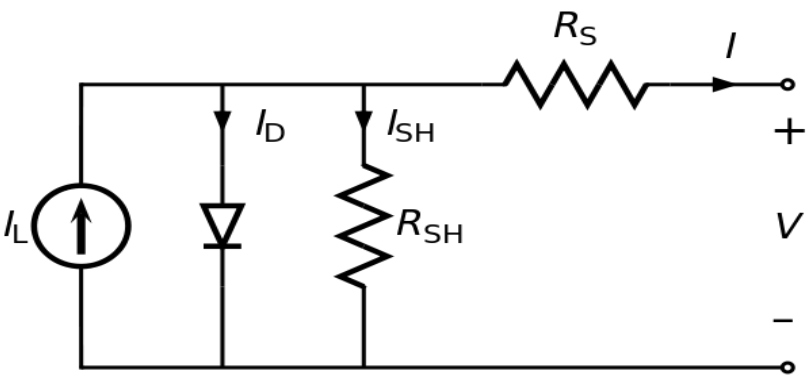

Fig 1

From the equivalent circuit it is evident that the current produced by the solar cell is [11] [12]:

$$
\mathrm{I}=\mathrm{I}_{\mathrm{L}}-\mathrm{I}_{\mathrm{D}}-\mathrm{I}_{\mathrm{SH}}
$$

where, $\mathrm{I}=$ generated solar cell current, $\mathrm{I}_{\mathrm{L}}=$ photo generated current, $\mathrm{I}_{\mathrm{D}}=$ diode current and $\mathrm{I}_{\mathrm{SH}}=$ shunt current.

The current through these elements is governed by the voltage across them:

$$
\mathrm{V}_{\mathrm{j}}=\mathrm{V}+\mathrm{IR}_{\mathrm{S}}
$$

where, $\mathrm{Vj}=$ voltage across both diode and resistor $\mathrm{R}_{\mathrm{SH}}, \mathrm{V}=$ voltage across the output terminals, $\mathrm{I}=$ output current and $\mathrm{R}_{\mathrm{S}}=$ series resistance.

According to Shockley diode equation [9], the current through the diode is

$$
\mathrm{I}_{\mathrm{D}}=\mathrm{I}_{0}\left(\mathrm{e}^{\left[\mathrm{V}_{\mathrm{j}} / \mathrm{nV} \mathrm{T}\right]}-1\right)
$$

where, $\mathrm{I}_{\mathrm{O}}=$ reverse saturation current, $\mathrm{n}=$ diode ideality factor. $\mathrm{V}_{\mathrm{T}}$ is the thermal voltage and $\mathrm{V}_{\mathrm{T}} \approx 0.0259$ volt at $25^{\circ} \mathrm{C}$. By Ohm's law, the current through the shunt resistor is:

$$
\mathrm{I}_{\mathrm{SH}}=\frac{\mathrm{V}_{\mathrm{j}}}{\mathrm{R}_{\mathrm{SH}}} \ldots
$$

where, $\mathrm{R}_{\mathrm{SH}}=$ shunt resistance. Substituting equation number $1 \& 3$ into the equation number 2 produces the characteristic equation of a solar cell

$$
I=I_{L}-I_{0}\left\{e^{\left[\frac{V+I R_{S}}{\eta V_{T}}\right]}-1\right\}-\frac{V+I_{S H}}{R_{S H}} \ldots . .
$$

When $\mathrm{R}_{\mathrm{S}}$ is not zero, the above equation does not give the current I directly, but it can then be solved using the Lambert W function:

$$
I=\frac{\left(I_{L}+I_{0}\right)-V / R_{S H}}{1+R / R_{S H}}-\frac{\eta V_{T}}{R_{S}} W\left(\frac{I_{0} R_{S}}{\eta V_{T}\left(1+R / R_{S H}\right)} e^{\left(\frac{V}{\eta V_{T}}\left(1-\frac{R_{S}}{R_{S}+R_{S H}}\right)+\frac{\left(I_{L}+I_{0}\right) R_{S}}{\eta V_{T}\left(1+R_{S} / R_{S H}\right)}\right)}\right)
$$

When $R_{S H}$ is infinite there is a solution for $V$ for any I less than $I_{L}+I_{0}$ :

$$
\mathrm{V}=\eta \mathrm{V}_{\mathrm{T}} \ln \left(\frac{\mathrm{I}_{\mathrm{L}}-\mathrm{I}}{\mathrm{I}_{0}}+1\right)-\mathrm{IR}_{\mathrm{S}}
$$


B. Output Characteristics of the Photo-voltaic Panel

When the cell is operated at open circuit $(I=0)$ then the voltage across the output terminals is defined as the opencircuit voltage. Assuming the shunt resistance is high enough to neglect the final term of the characteristic equation, the open-circuit voltage $\mathrm{V}_{\mathrm{OC}}$ is:

$V_{O C} \approx \frac{n k T}{q} \ln \left(\frac{I_{L}}{I_{0}}+1\right)$

Similarly, when the cell is operated at short circuit, the current I through the terminals is defined as the short-circuit current. The short-circuit current $\mathrm{I}_{\mathrm{SC}}$ is:

$I_{S C} \quad I_{L}$

It is not possible to extract any power from the device when operating at either open circuit or short circuit conditions. The output characteristics of the photovoltaic is shown in fig 2

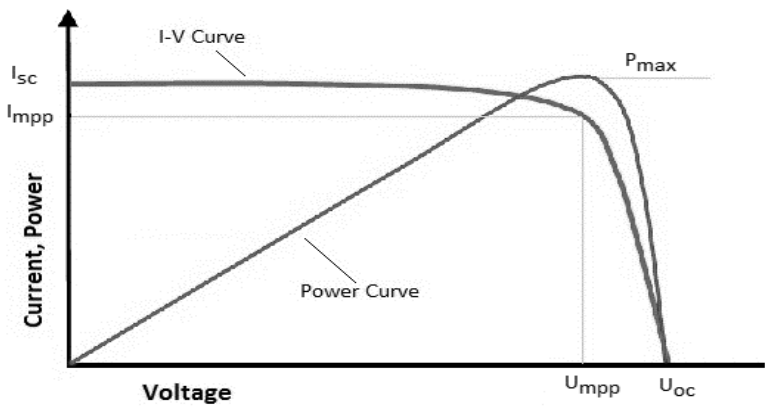

Fig 2

C. Simulation model of the Supercapacitor

Supercapacitor are usually double layered capacitor or DLC's and typically have very high capacitance value allowing them to store large quantities of electrical energy but the voltage remains low (maximum 3V) [8]. So, it helps to drive motor, pump and other electro-mechanical devices which requires impulsive power. The energy stored in a Supercapacitor is the product of half of the capacitance and the square of voltage. Thus the high capacitance value is the distinguishing factor as the voltage which is fed to the system is almost constant for efficient charging and Supercapacitors can charge/ discharge rapidly. Its small leakage current helps in long period of energy storage and the efficiency could exceed $95 \%$.

In this paper we have used two resistance model of the Supercapacitor for software simulation as shown in fig 3.

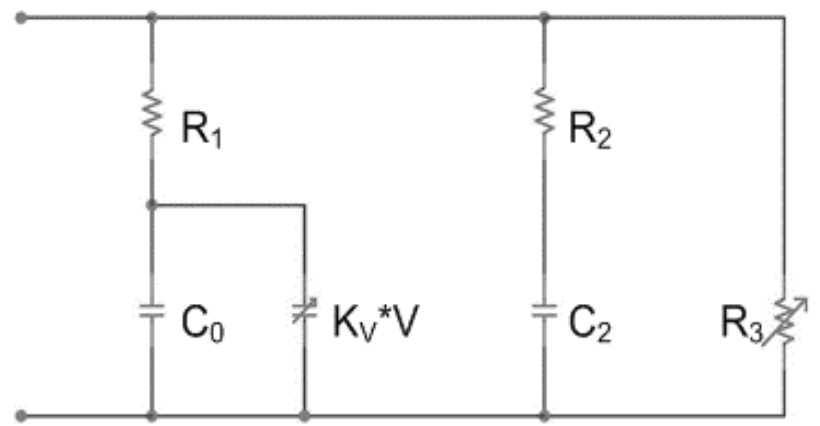

Fig. 3

Here $C_{0}$ is a constant capacitor, $R_{1}$ is the equivalent series resistance and $K_{V} * V$ is a voltage dependent capacitor and this first branch models the voltage dependencies of the Supercapacitor. The second branch consisting of $R_{2}$ and $C_{2}$ are responsible for taking into account the charge redistribution taking place within the Supercapacitor. Theresistance $\mathrm{R}_{3}$ is called the equivalent parallel resistor and is used to model the leakage behaviour of capacitor. The Supercapacitor has an inherent tendency of reduction in storage efficiency with increase in the value of ESR and the slope of the curve changes with higher values of ESR. The storage efficiency is also a function of capacitance and with increasing value of capacitance the storage efficiency of the Supercapacitor increases. The slope of the curve keeps on decreasing as the capacitance reaches higher and higher values. 
D. Simulation model of the buck Converter

The buck converter (shown in fig 4) or step down converter is basically a switched mode DC-DC power converter which can transfer packets of energy from its input to output depending on the frequency of the switching signal applied to the switch $\mathrm{S}$, which in most cases is usually a power transistor such as MOSFET whose gate is used to control the switching action. Buck converters in most cases could be very efficient and can provide efficiencies more than $90 \%$.Simulink model of the buck converter used in our simulation is shown in fig 5.

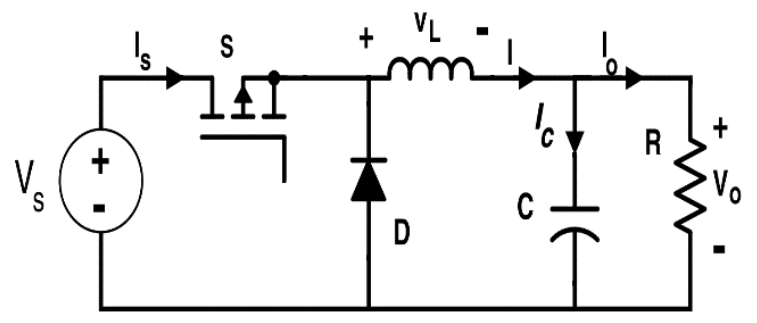

Fig 4

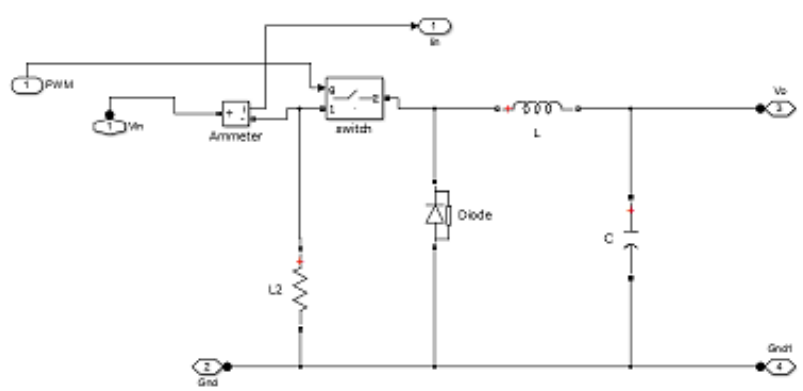

Fig 5

E. Application of Maximum Power Point Tracking for efficient power delivery to load

In our simulation to maximise the power delivery from PV panel to the Supercapacitor we have used the MPPT. Maximum power point tracking algorithm is a tracking algorithm usually employed to maximize the output power to loads from photovoltaic panels. Different MPPT algorithms such as Perturb and Observe, Incremental Conductance etc. try to find out the point from the P-V characteristics of the solar panel where the power delivered by the panel is maximum. Out of many available MPPT algorithms,Perturb and Observe algorithm appears to be most promising with better tracking efficiency. The $\mathrm{P} \& \mathrm{O}$ algorithm is shown in the flowchart below.

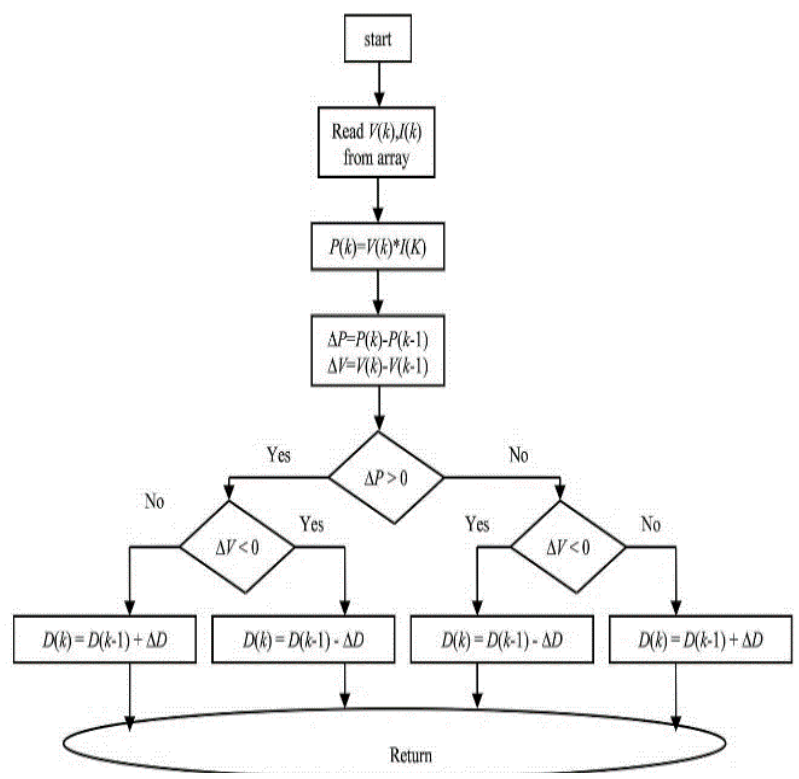

Simulink model of the Perturb and Observe algorithm used is as shown in fig 6. 


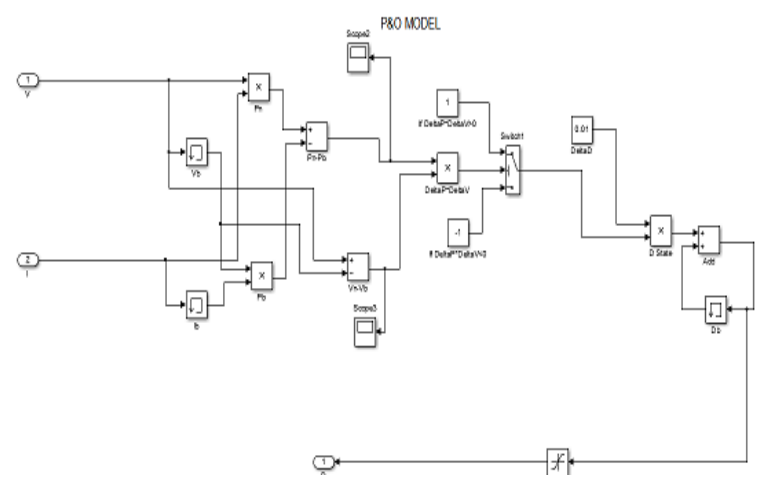

Fig 6

F. Complete simulation setup of the system

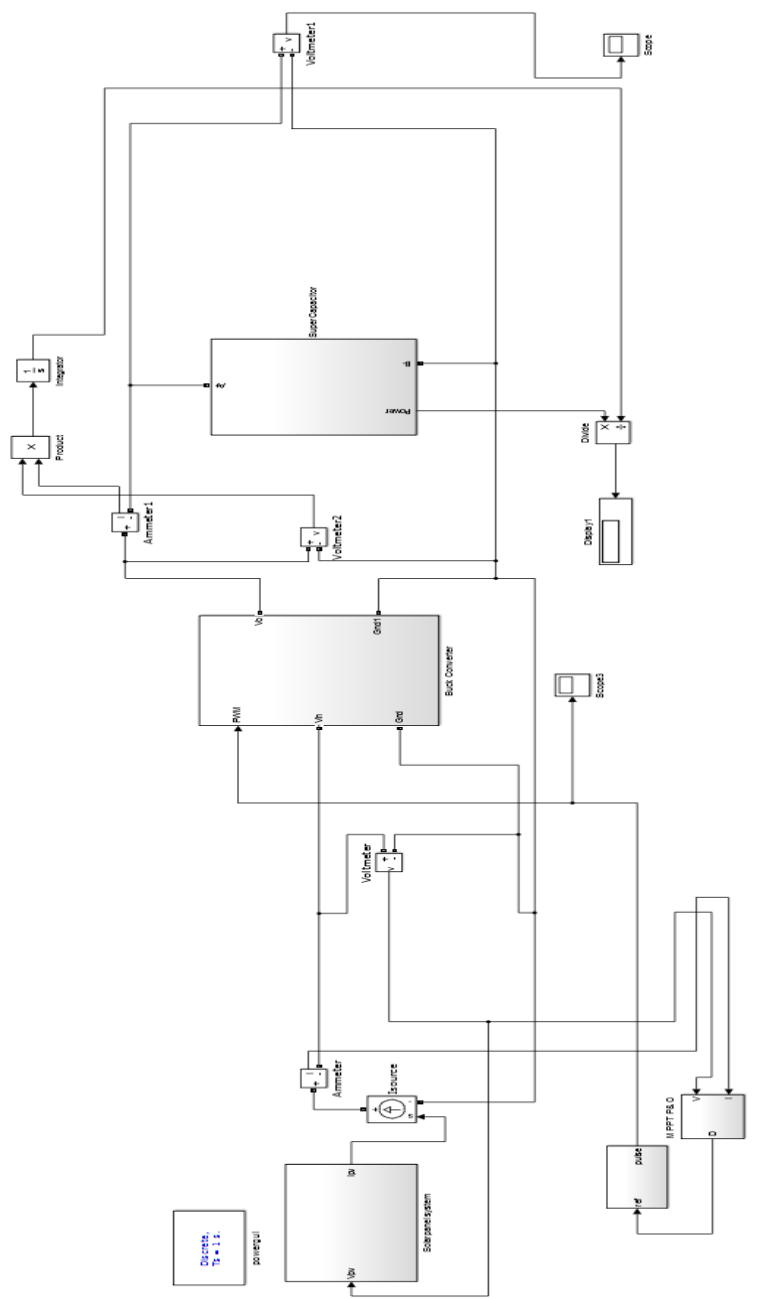

Fig 7

\section{G. Genetic Algorithm}

Genetic algorithm (GA) is an artificial search algorithm based on principles of natural selection and natural genetics. It is a programming technique that imitates biological evolution as a problem-solving strategy [7] [10]. GA represents a popular approach to optimization because it selects the best solutions among multiple local minima. The GA assigns the present population as parents and uses them to produce the children for the future generation. As generation increases the population evolves toward an optimal solution.

Sometimes the optimal solution is achieved by employing a certain 'fitness function' which takes a candidate solution to the problem as input and produce output as how fit the solution is with respect to that problem. A fitness function should be fast to compute and it should measure quantitatively how fit the given solution is to the given problem. 
ISO 3297:2007 Certified

Vol. 4, Issue 5, May 2017

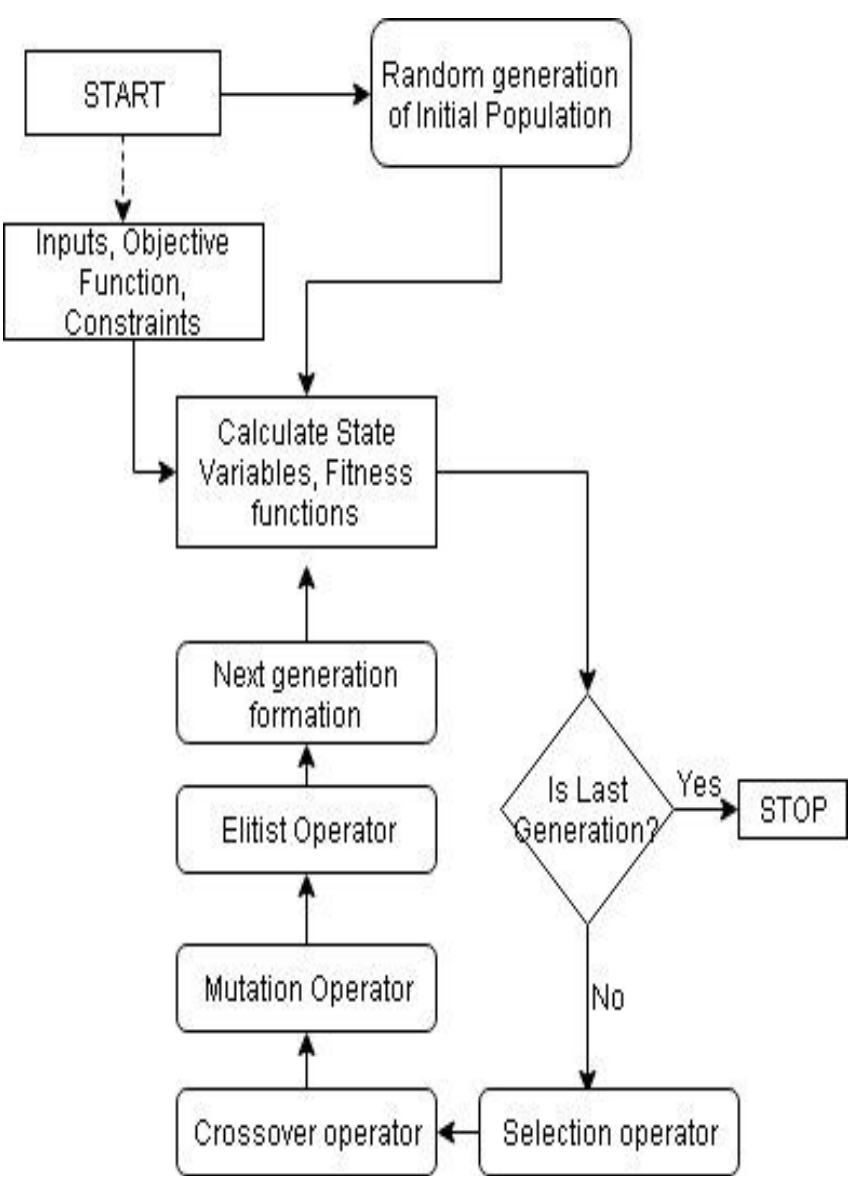

\section{III.VERIFICATION OF THE VARIATION IN STORAGE EFFICIENCY WITH ESR AND CAPACITANCE}

We have simulated the model and verified the storage efficiency variations with variation in ESR and capacitance. The storage efficiency vs. ESR response obtained from the model is shown in fig 8.

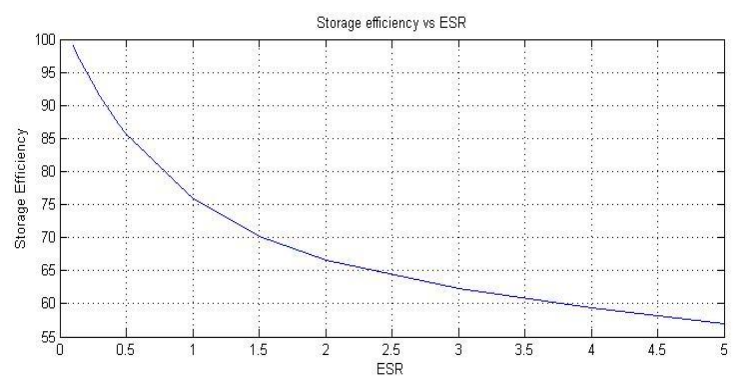

Fig 8

The storage efficiency vs. Capacitance response obtained from the model is shown in fig 9.

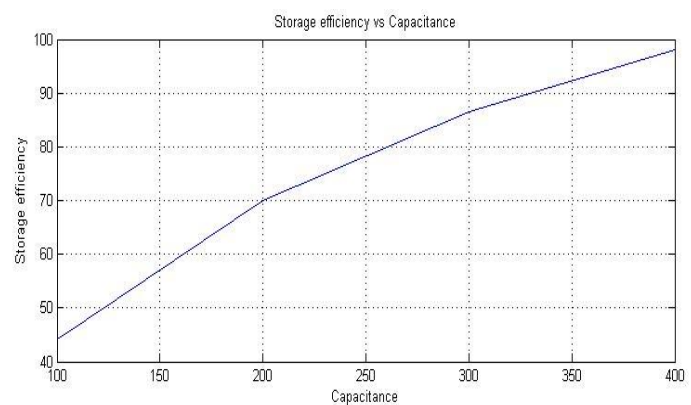

Fig 9 


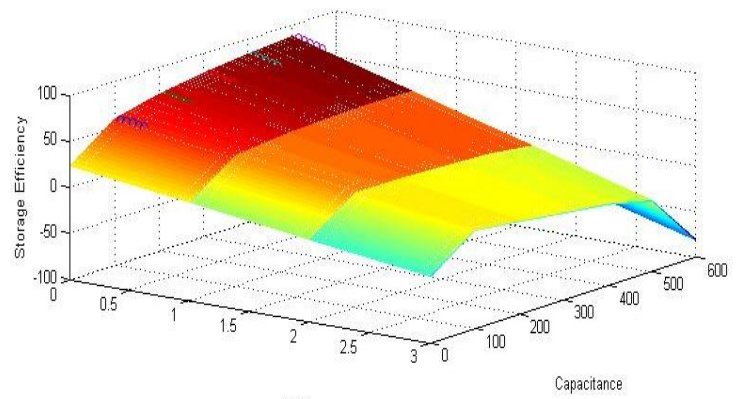

Fig 10

The 3D plot of efficiency vs. ESR vs. Capacitance obtained after extrapolation of the sample data obtained from the model is shown in fig 10.

\section{IV.EXPERIMENTAL RESULTS}

\begin{tabular}{|c|c|c|}
\hline \multicolumn{3}{|c|}{ Table 1} \\
\hline ESR(ohm) & Capacitance (Farad) & Storage efficiency (\%) \\
\hline \multirow{5}{*}{0.05} & 100 & 58.63 \\
\hline & 200 & 69.26 \\
\hline & 300 & 76.83 \\
\hline & 400 & 81.37 \\
\hline & 500 & 85.06 \\
\hline \multirow{5}{*}{0.1} & 100 & 57.45 \\
\hline & 200 & 67.88 \\
\hline & 300 & 74.69 \\
\hline & 400 & 79.45 \\
\hline & 500 & 82.96 \\
\hline \multirow{5}{*}{0.15} & 100 & 56.52 \\
\hline & 200 & 66.58 \\
\hline & 300 & 73.11 \\
\hline & 400 & 77.66 \\
\hline & 500 & 81 \\
\hline \multirow{5}{*}{0.2} & 100 & 55.64 \\
\hline & 200 & 65.37 \\
\hline & 300 & 71.64 \\
\hline & 400 & 75.99 \\
\hline & 500 & 79.18 \\
\hline \multirow{5}{*}{0.25} & 100 & 54.82 \\
\hline & 200 & 64.22 \\
\hline & 300 & 70.26 \\
\hline & 400 & 74.43 \\
\hline & 500 & 77.48 \\
\hline
\end{tabular}

The simulation of the model was done to get enough sample points for further processing by the Genetic algorithm. At first the model was used to determine the variation in efficiency with variation in ESR and Capacitance individually and independently and the data points were plotted as shown in the graphs in Part III. These sample data points were then used to find the polynomial function which will eventually be modified and used as a fitness function in Genetic Algorithm. The data points observed from the model are given in the table 1.

Further this data have been used to get the polynomial function using the Curve fitting toolbox of MATLAB.

The obtained polynomial is of order 2 and is given as:

where, $\mathrm{x}$ is the equivalent series resistance (ESR) and $\mathrm{y}$ is the capacitance of the Supercapacitor,

$$
z(x, y)=p_{00}+p_{10} x+p_{01} y+p_{20} x^{2}+p_{11} x y+p_{02} y^{2}
$$

$\mathrm{p} 00=47.79, \mathrm{p} 10=-20.41, \mathrm{p} 01=0.1339, \mathrm{p} 20=19.6$,

$\mathrm{p} 11=-0.04914$ and $\mathrm{p} 02=-0.0001104$. 


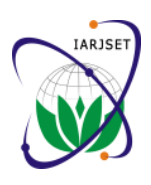

\section{IARJSET

We have used this polynomial in genetic algorithm for optimization of the efficiency since the genetic algorithm tool in MATLAB is configured for data minimization we have used fitness function $f(x, y)$,so that minimization of $f(x, y)$ will lead us to maximization of $\mathrm{z}(\mathrm{x}, \mathrm{y})$ which is essentially the overall efficiency.

$$
f(x, y)=1 / z(x, y)
$$

Final data obtained after optimization by the Genetic algorithm at different stall generations is as follows:

\begin{tabular}{|l|l|l|l|}
\hline STALL GENERATION & ESR (ohm) & CAPAC-ITANCE (Farad) & EFFICIENCY (\%) \\
\hline 20 & $1.00 \mathrm{E}-003$ & 498 & 86.86 \\
\hline 21 & $3.00 \mathrm{E}-003$ & 473 & 86.3 \\
\hline 22 & 0 & 458.15 & 85.95 \\
\hline 23 & $2.30 \mathrm{E}-002$ & 432.1 & 84.09 \\
\hline 24 & $1.20 \mathrm{E}-002$ & 414.35 & 83.82 \\
\hline 25 & $1.60 \mathrm{E}-002$ & 479.79 & 85.9 \\
\hline
\end{tabular}

\section{CONCLUSION}

In conclusion, it should be clearly emphasised that a lower value of ESR and high Capacitance is desired for better storage efficiency. It can be observed from the shown graphs that storage efficiency increases with decrease in ESR and increase in capacitance of Supercapacitor.

The initial results shows that typical storage of efficiencies greater than $85 \%$ can be obtained at the above obtained values of ESR and capacitance, which clearly is an optimized one. This method explores the area of implementation of AI computing techniques in optimization of Supercapacitor performance. This method can be used to determine optimum values of ESR and capacitance for practical applications.

\section{REFERENCES}

[1] T.M. Razykov, C.S. Ferekides, D.Morel, E.Stefanakos, H.S Ullal, H.M. Upadhayaya, "Solar photovoltaic electricity: Current status and future prospects", Elsevier, Volume 85, Issue 8, August 2011, Pages 1580 - 1608.

[2] Mikio Taguchi, Ayumu Yano, Satoshi Tohoda, Kenta Matsuyama, Yuya Nakamura, Takeshi Nishiwaki, Kazunori Fujita, Eiji Maruyama, IEEE Journal of Photovoltaics, "24.7\% Record Efficiency HIT Solar Cell on Thin Silicon Wafer", Volume:4, Issue: 1, Jan.2014, Pages 96-99.

[3] N. Femia, G. Petrone, G. Spagnulo, M. Vitelli, "Optimization of perturb and observe maximum power point tracking method", IEEE Transactions on Power Electronics, Volume: 20, Issue: 4, July 2005, Pages: 963-973.

[4] E. Koutroulis, K. Kalaitzakis, N.C. Voulgaris, "Development of a microcontroller-based, photovoltaic maximum power point tracking control system", IEEE Transactions on Power Electronics, Volume: 16, Issue: 1, Jan 2001, Pages: 46-54.

[5] Nan Li, Jiancheng Zhang, Yun Zhong, "A Novel Charging Control Scheme for Supercapacitor Energy Storage in Photovoltaic Genera tion System", Third International Conference on Electric Utility Deregulation and Restructuring and Power Technologies, 2008. DRPT 2008.

[6] UmaShankar Patel, Ms. Dhaneshwari Sahu, Deepkiran Tirkey, "Maximum Power Point Tracking Using Perturb \& Observe Algorithm and Compare With another Algorithm", International Journal of Digital Application \& Contemporary research, Volume 2, Issue 2, September, 2013.

[7] Richa Garg, Saurabh Mittal, "Optimization by Genetic Algorithm”, International Journal of Advanced Research in Computer Science and Software Engineering, Volume 4, Issue 4, April 2014.

[8] R. Faranda, M. Gallina and D.T. Son, "A new simplified model of Double-Layer Capacitors”, International Conference on Clean Electrical Power, 2007. ICCEP' 07.

[9] W. Shockley, "The Theory of p-n Junctions in Semiconductors and p-n Junction Transistors", Bell Labs Technical Journal, Volume 28, Issue 3, July 1949.

[10] David E. Goldberg, "Genetic Algorithms in search, Optimization and Machine learning", Pearson India (2002).

[11] Antonio Luque \& Steven Hegedus, "Handbook of Photovoltaic Science and Engineering”, John Wiley and Sons, 2003, ISBN 0-471-49196-9.

[12] Jenny Nelson, "The Physics of Solar Cells", Imperial College Press, 2003, ISBN 978-1-86094-340-9.

[13] Lisheng Shi and M. L. Crow; "Comparison of Ultracapacitor Electric Circuit Models", IEEE Conf. "Power and Energy Society General Meeting - Conversion and Delivery of Electrical Energy in the 21st Century", Aug. 2008.

[14] Tanusree Dutta, Rabindranath Ghosh, Indranil Das,Hiranmay Saha, "An Analytical Approach to Charging Characteristics of Supercapacitors for Different Equivalent Circuits using DC and Photovoltaic Sources", International Advanced Research Journal in Science, Engineering and Technology, Vol.3, Issue 7, July 2016. 